\title{
INFLUENCE OF END POTENTIAL PLATES ON PLASMA HEATING AND CONFINEMENT
}

\author{
Sergey Yu. Taskaev \\ Budker Institute of Nuclear Physics \\ avenue Lavrent'ev, 11 \\ 630090 Novosibirsk, Russia \\ $+7(3832) 359550$
}

\section{ABSTRACT}

Hot target plasma was obtained in AMBAL-M open trap. In the present work, transverse current at plasma periphery, peculiarities of longitudinal current flow in plasma, and energy balance of electrons in a mirror were studied.

The consideration allowed to find out the mechanism of electron heating up to high temperature. It was detected that this «non-joule» non-turbulent effective heating of electrons was related to the current flow and the experiment features.

\section{INTRODUCTION}

Hot target plasma was obtained without additional heating in an open trap AMBAL-M from arc source (gun) located behind the mirror [1]. The plasma has a diameter of $20 \mathrm{~cm}$, an average density of $6 \times 10^{12} \mathrm{~cm}^{-3}$, an ion energy of $200 \mathrm{eV}$, and high electron temperature of $50 \mathrm{eV}$ that is of the most importance. It is just the high value of electron temperature that rises the question about the mechanism of its keeping up. According to the Pastukhov's formula [2], the energy losses of the trapped electrons from the mirror are estimated to be no less than $20 \mathrm{~kW}$. The obvious classical collision processes electron heating from the more hot ions $(3 \mathrm{~kW})$ and Joule heating concerning Spitzer conductivity $(1 \mathrm{~kW})$ - are not enough for balance. Does that mean that the mechanism of electron heating is turbulent or collision, but not obvious at ordinary estimations?

In the present work, the processes of plasma heating and confinement are investigated. Particular attention is given to the peculiarities of this experiment.

After a short description of the experimental layout and the scenario of obtaining of plasma in the trap, the transverse ion current flow will be first considered at the periphery in the transporting region, which is necessary to close the circuit. Then, the feature of revealed longitudinal electron current flow from transporting region into the mirror will be considered which is related to the presence of electric field accelerating electrons. Further a possibility will be shown to form the flow of fast electrons transporting the current. The results of measurements of electron energy distribution function in the mirror will be presented. Next, heating of the trapped electrons by the flow of fast electrons will be considered, and its efficiency will be shown. In conclusion the main results of the work will be formulated.

Note that end potential plates in this very experiment mean potential electrodes of a plasma gun.

\section{EXPERIMENTAL LAYOUT}

In Fig. 1 the experiment scheme is shown. The plasma is generated in $2 \mathrm{~ms}$ by the arc source [3] located behind the mirror. The gun generates a narrow ring dense plasma jet. The inner diameter of the jet at the output of the gun is $11 \mathrm{~cm}$, the outer is $13 \mathrm{~cm}$. Another feature of the source is

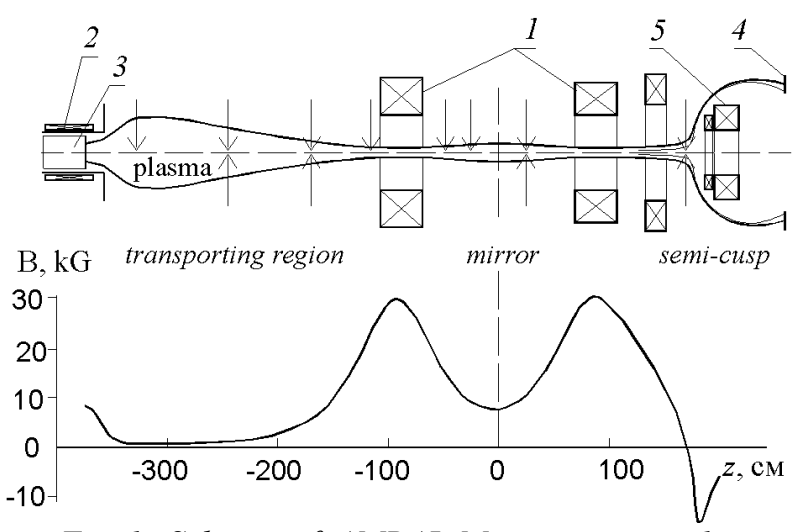

Fig. 1. Scheme of AMBAL-M experiments. 1mirror coils, 2 - gun solenoid, 3 - plasma gun, 4 - plasma receiver, 5 - semi-cusp coil. The arrows from above show the coordinates of the cross sections where Langmuir probes were measured, from below - magnetic probes. 
that the arc source electrode potentials form a nonequilibrium radial electric field (Fig. 2). The nonuniform azimuthal drift causes the Kelvin-Helmholtz instability [4]. The development of the instability is accompanied by frequency modulation of azimuthal electric field and stochastic heating of magnetized ions into the transverse degree of freedom [5].

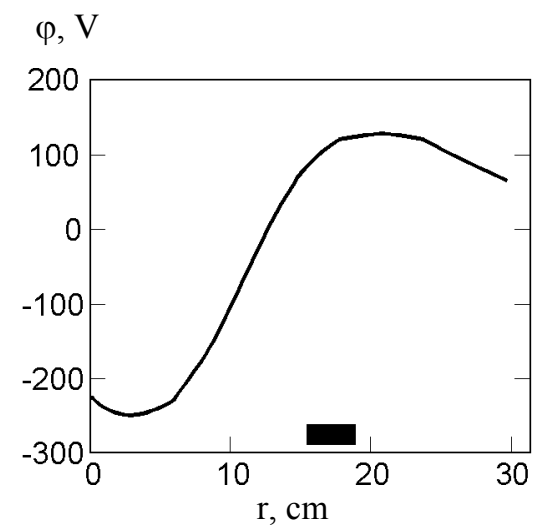

Fig. 2. Radial profile of plasma potential in the transport region $(z=-243 \mathrm{~cm})$. Position of the gas-discharge channel is marked with the bold line segment.

On moving to the mirror, the ions get heated, and the free path increases. The major part of the plasma flow is reflected, and plasma density decreases, and the plasma potential also decreases. A thermobarrier in the throat is realized $[6,1]$. Ions in the mirror are kept by magnetic field, electrons - by magnetic field and ambipolar potential.

Longitudinal electron current of $1 \mathrm{kA}$ in the plasma was found and investigated [7]. In this paper, we shall first concentrate on the transverse ion current necessary for closing the circuit, clear up the mechanisms of its flowing, and then demonstrate the peculiarity of longitudinal electron current flow.

\section{TRANSVERSE ION CURRENT}

Assume that plasma is magnetized and it takes certain volume in the transporting region. Let us consider processes resulting in occurrence of transverse current through the surface which separates the body of plasma (contacting with the isolated gun at the left) from the outer volume of plasma (contacting with ground at the left). Choosing this surface is determined by the possibility to exclude the effect of shorting by conducting ends due to boundary conditions (the Simon's effect [8]). The longitudinal transfer from the inner volume through the right boundary, or the mirror entrance, depends on this transverse current. The plasma density decreases outward $(\partial \mathrm{n} / \partial \mathrm{r}<0)$ and electric field is directed outward $\left(\mathrm{E}_{\mathrm{r}}>0\right)$ on this separating surface.

The feature of the experiment is a narrow plasma jet. Drift in the cross fluctuating azimuthal electric field and the leading magnetic field, and ion-ion collisions result in the widening of the jet. The second process, in contrast to the first one, leads also to the considerable radial current. This process of widening of the narrow plasma jet may be described by a diffusion ratio, but it can hardly be named diffusion in a narrow sense. This current value can be estimated in the following way:

$$
I_{\perp}=\int 0.5 \frac{e \rho_{i}^{2}}{\tau_{i i}} \frac{\partial n}{\partial r} 2 \pi r d z \propto S \frac{e v_{T_{i}}^{2}}{2 \omega_{c i}^{2} \tau_{i i}} \frac{n}{L_{\perp}}=280 \mathrm{~A} .
$$

The big value of current is appreciably determined by big square of the limiting surface $\left(3.7 \times 10^{4} \mathrm{~cm}^{2}\right)$ and big ratio of Larmor radius to the density change distance $\rho_{\mathrm{i}} / \mathrm{L}_{\perp} \sim 1 / 4$. To illustrate another main reason of this strong transverse current, let us consider a rough model of the transporting region as a big mirror, and a gun generates a plasma jet of $\mathrm{I}_{\mathrm{pl}}$ into it. Denote the plasma lifetime in the mirror as $\tau_{\text {life }}$. Then, plasma density is $\mathrm{n}=\mathrm{I}_{\mathrm{pl}} \tau_{\text {life }} / \mathrm{er}^{2} \mathrm{~L}_{\|}$; taking into account that $\tau_{\mathrm{ii}} \propto n^{-1}$, we may have the following ratio of the transverse current

$I_{\perp} \propto S \frac{n^{2}}{B^{2} L_{\perp}} \propto I_{p l}^{2} \tau_{\text {life }}^{2} \frac{r}{L_{\|} L_{\perp}}$. The transporting region is intended for transportation of plasma jet into the main mirror. We accomplished the transportation of the whole plasma jet generated by the gun by realization of collision subsonic flow [9]. The lifetime $\tau_{\text {life }}$ was equal to the flow time for the region with the ion velocity, and the longitudinal current was negligible. In this very case, through sufficiently fast rising of the magnetic field and ion heating into transverse degree of freedom [5] collisionless flow is realized in front of the throat, and the major part of the flow $(\sim 0.9 \div 0.95)$ becomes reflected by the increasing magnetic field. The plasma lifetime $\tau_{\text {life }}$ in the transporting region increases considerably from the previous case. As the longitudinal confinement of plasma is improved than the transverse losses become important.

As there is no ban for both longitudinal current (due to the contact with the system's end of conducting vacuum chamber), and Hall's current (due to plasma cylindricity), the conductivity current $j_{\perp}=\frac{\sigma_{\perp}}{1+\left(\omega_{\mathrm{ce}} \tau_{\mathrm{e}}\right)^{2}} E_{\perp}$ flows along the direction of the radial electric field applied, and the current is suppressed significantly by strong magnetic field. The current is

$$
I_{\perp}^{E}=\int \frac{\sigma_{\perp} E_{\perp}}{\left(\omega_{c e} \tau_{e}\right)^{2}} 2 \pi r d z \propto S \frac{e^{2} n}{m \omega_{c e}^{2} \tau_{e}} E_{\perp}=200 \mathrm{~A} .
$$

Note that even small heterogeneity in conductivity of strongly magnetized plasma hinders the Hall's current flow and may cause a considerable increase in current along the 
radial electric field. This effect was reported in [10] and then studied in [11]. This turbulent current value can be estimated in the following way. Collision processes lead to the current of $480 \grave{A}$, which is known with the accuracy of 2 due to the accuracy of plasma parameter measurement. Therefore, heterogeneity in conductivity may both play no role and lead to the increase in current along the radial electric field of the order of collision current. So, one can conclude that there is a small, if any, influence of turbulence on the transverse current flow.

To understand these two collision processes, let us advance a simple consideration. Ions are more mobile at the cross direction, and to keep them from going outward a negative radial electric field is to be established. We have a positive one, that, on the contrary, favours the transverse ion flow outward. Change of direction of radial electric field at periphery results in considerable decrease in transverse current. That was confirmed by the experiments specially conducted, and that correlates with the understanding of the processes.

To complete the picture, it should be noticed that starting with the time of $1.2 \mathrm{~ms}$, the longitudinal current flows from the extending outward grounded frame of the solenoid to the anode of the gun. The contribution into the transverse current of the following processes appeared to be quite small. These processes are: collisions with neutral particles, ambipolar diffusion of plasma in space with not uniform potential, magnetic turbulent transport $u_{\|}\left\langle\widetilde{n} \widetilde{B}_{r}\right\rangle / B$. The last one was experimentally determined with the use of nearby small Rogowskii coil and Langmuir probe [12].

So, the big value of the current is explained by the non-equilibrium radial electric field in plasma, given by the arc gun electrode potentials, small transverse gradient value of the density change due to the narrow jet, big square of plasma surface due to the weak magnetic field, and improved longitudinal confinement of plasma in the transporting region.

\section{LONGITUDINAL ELECTRON CURRENT}

As ions flow outside in the transporting area, and there is no runoff to an isolated gun, the electrons flow out into the mirror along the field to provide quasineutrality. The current shorting is realized by a conducting vacuum chamber. Longitudinal electron current of $1 \mathrm{kA}$ in the plasma was found and investigated [7]. The current was determined to be a part of the gun arc current [13]. When coming to the mirror the longitudinal current becomes displaced to the center and flows mainly along the magnetic field force lines with radii of about $1 / 2$ the gas discharge channel radius. The plasma potential was found to increase on these force lines when coming closer to the mirror and the thermobarrier was not found in the throat area (Fig. 3). This field formation could be possibly related to potential non-equilibrium cross profile as a result of the transverse diffusion of plasma. The need for longitudinal electron current flowing in the area of low plasma density could also cause the field formation. The potential increases $150 \mathrm{~V}$ on the length of $2 \mathrm{~m}$.

\section{FAST ELECTRON FLOW FORMATION}

It is clear that a collisionless acceleration of electrons with initial temperature of $10 \mathrm{eV}$ by this electric field in a homogeneous magnetic field could cause formation of almost monochromatic electron beam with longitudinal energy of $150 \mathrm{eV}$. The solving of Vlasov's equation shows that the presence of increasing magnetic field leads to the electron spectrum widening at longitudinal energy. The cause is the transition of energy from longitudinal to transverse due to adiabatic invariant conservation. The absence of sharp boundary of electron runaway in the accelerating electric field could result in even the more significant widening of the electron spectrum. When coming closer to the mirror the plasma density decreased, the boundary of the runaway area is shifted to the area of the lower velocities, and the more electrons become involved into the runaway process. The fast electron beam relaxation to the plato state is also possible due to development of Langmuir instability.

Therefore, the longitudinal electric field found forms the population of fast electrons which transports the current in the mirror. The fast electron flow in the mirror was experimentally registered [14] by electron energy analyzer (a probe with small inlet and electrostatic repelled lens). The diagram of the function of electron distribution over the longitudinal energy looks like a plato in the energy range of $150 \div 300 \mathrm{eV}$.

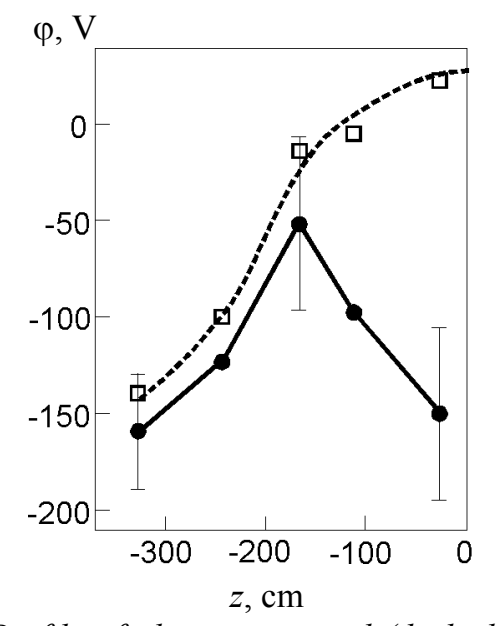

Fig. 3. Profile of plasma potential (dashed line) and floating potential of probe (solid line) along a magnetic field line on which the significant longitudinal electron current flows. 


\section{ELECTRON HEATING BY FAST ELECTRON FLOW}

In case of current being transferred by not all electrons but the small population of fast electrons, the electron heating by the current is stronger.

That can be easily shown qualitatively. Let the current be transferred by all electrons. Their density is equal to $\mathrm{n}$, the flow velocity $-\mathrm{u}$, heat (chaotic) velocity $\mathrm{v}_{\mathrm{T}}$, and $\mathrm{u}<<\mathrm{v}_{\mathrm{T}}$. In a time $\tau$ determined by the velocity of the relative movement, i.e. heat velocity, the electrons lose the longitudinal momentum nmu. Friction force is $n m u / \tau$ and the power is $P_{\text {joule }}=n m u^{2} / \tau \sim j^{2} / n \tau \sim 1 / n$, where $j=$ enu. Let the same current be now transferred by the part of electrons with the density of $\mathrm{n}_{\mathrm{f}}\left(\mathrm{n}_{\mathrm{f}}<<\mathrm{n}\right)$ and the velocity of $\mathrm{u}_{\mathrm{f}}\left(\mathrm{u}<<\mathrm{u}_{\mathrm{f}} \sim \mathrm{v}_{\mathrm{T}}\right)$. The current electrons lose the same longitudinal momentum $\mathrm{n}_{\mathrm{f}} \mathrm{mu}_{\mathrm{f}}$ in the same time interval $\tau$. The friction force is the same $-\mathrm{n}_{\mathrm{f}} \mathrm{mu}_{\mathrm{f}} / \tau$, but the power generated increases considerably $\mathrm{P}=\mathrm{n}_{\mathrm{f}} \mathrm{mv}_{\mathrm{f}}^{2} / \tau \sim \mathrm{j}^{2} / \mathrm{n}_{\mathrm{f}} \tau \sim$ $\mathrm{P}_{\text {joule }} \mathrm{n} / \mathrm{n}_{\mathrm{f}}$.

Besides, to achieve the more precise understanding of mechanism of heating, one can consider the flow of fast electrons with the energy of $\varepsilon=100 \mathrm{eV}$ in an equilibrium plasma with the temperature of $\mathrm{T}=50 \mathrm{eV}$ [15]. The time of their passing the mirror is $\tau_{\mathrm{fl}}=0.3 \mu \mathrm{s}$. The characteristic collision time is $\tau_{1}^{e}=\frac{\sqrt{m}}{\pi \sqrt{2} e^{4}} \frac{\varepsilon^{3 / 2}}{\lambda n} \approx 3 \mu \mathrm{s}$. While flying through the mirror, the fast electrons lose their momentum and transfer the energy due to the collisions with ions and electrons kept in the mirror. The time of momentum loss $\tau_{\mathrm{s}}$ and energy transfer time $\tau_{\varepsilon}$ are shown in the Table.

\begin{tabular}{|l|c|c|}
\hline & ions & electrons \\
\hline$\tau_{\mathrm{s}}$ & $\tau_{1}$ & $\approx \tau_{1} / 1.4$ \\
\hline$\tau_{\varepsilon}$ & $>>\tau_{1}$ & $\approx \tau_{1}$ \\
\hline
\end{tabular}

Fast electrons at collisions with ions lose their momentum only, and at collisions with trapped electrons lose the momentum and transfer the energy if $\varepsilon \geq 1.5 \mathrm{~T}_{\mathrm{e}}$. The loss of momentum results in the raising of fast electrons temperature $13 \mathrm{eV}$ (the power may be estimated as $\mathrm{Q}=\mathrm{I} \varepsilon \tau_{\mathrm{fl}} / \tau_{\mathrm{s}} \approx 20 \mathrm{~kW}$ ). Energy transfer leads to heating of the trapped electrons. Though the heating power is lower $\left(\mathrm{Q}=\mathrm{I} \varepsilon \tau_{\mathrm{f}} / \tau_{\varepsilon} \approx 10 \mathrm{~kW}\right)$, the trapped electrons are heated to higher temperature, as the lifetime of the trapped electrons in the mirror is much longer than the lifetime of the flying ones. So, the mechanism of the trapped electron heating is the energy transfer from the fast electrons carrying the current to the trapped electrons as a result of Coulomb collisions.

The precise calculations confirm significant heating of electrons kept in the mirror by the fast electron flow which carries the current. This heating mechanism allows to close the energy balance. The fast electron flow heats the trapped electrons effectively (due to an ambipolar potential and high velocity of the flow electrons) as a result of Coulomb collisions.

Presence of strong current does not affect the hot ions lifetime as the fast electron density is negligibly low, and therefore the power transferred that is carried out quickly by the flow from the mirror is also low. Absence of thermobarrier in the throat does not affect the plasma parameters in the mirror. Though the potential minimum is far from the mirror in the transporting region, the electrons on these force lines still are in deep potential well. These force lines of the magnetic field are not projected into gasdischarge channel with high emission ability and low temperature.

\section{CONCLUSION}

Flow of transverse and longitudinal current in plasma, and energy balance of electrons in AMBAL-M open trap were studied. The mechanism of electron heating related to the current flow and the experiment peculiarities were detected.

It was cleared up that the features and peculiarities of the experiment led to:

- transverse ion current in transporting region;

- longitudinal electron current from transporting region into mirror;

- formation of electric field accelerating electrons at the outlet from transporting region;

- formation of fast electron flow which transfers the current in this electric field;

- effective heating of trapped electrons by fast electron flow as a result of Coulomb collisions.

Features of the experiment were the realization of thermobarrier and ambipolar potential.

It was stated that the peculiarities of the experiment playing a considerable role in the processes named were:

* in the magnetic vacuum system: conducting grounded vacuum chamber, and big area of low magnetic field,

* in the plasma gun: narrow ring jet that is generated by the gun; electrode potential form a non-equilibrium radial electric field in the plasma,

* improved longitudinal confinement of plasma in the transporting region.

«Non-joule» non-turbulent effective heating of electrons by the current has been first identified and investigated. The model described shows the possibility of obtaining of hot plasma in a mirror and the possibility of change in plasma confinement by control of the radial profile of the electric field using end potential electrodes. The plasma state obtained is close to the one which can be described basing on the classical (collisional) phenomena. It was determined that non-equilibrium profile of radial 
electric field set by the arc gun electrode potentials has the major influence on realization of this efficient heating mechanism. This control of the radial profile of the potential and the «classical» character of the plasma obtained are pointed out as characteristic favourable features of open traps.

\section{NOMENCLATURE}

$\begin{array}{ll}\mathrm{n} & \text { density } \\ \mathrm{E} & \text { electric field } \\ \mathrm{B} & \text { magnetic field } \\ \varepsilon & \text { energy } \\ \mathrm{I} & \text { current } \\ \mathrm{j} & \text { current density } \\ \mathrm{v} & \text { velocity } \\ \mathrm{u} & \text { flow velocity } \\ \mathrm{m} & \text { electron mass } \\ \tau & \text { collision time } \\ \rho & \text { Larmor radius } \\ \omega & \text { girofrequency } \\ \sigma & \text { conductivity }\end{array}$

\section{ACKNOWLEDGMENTS}

The author would like to thank G. I. Dimov, V. I. Davydenko, A. A. Kabantsev, and V. G. Sokolov for discussion. This research was sponsored in part by Russian Foundation for Basic Research, project no. 98-02-17801.

\section{REFERENCES}

1. T. D. Akhmetov, V. S. Belkin, E. D. Bender, et. al., "Production of a hot initial plasma in the end cell of the AMBAL-M device," Fiz. Plasmy, 23, 988 (1997) [Plasma Phys. Reports (Engl. Transl.), 23, 911 (1997)].

2. V. P. Pastukhov. Reviews of Plasma Physics, Moscow: Energoatomizdat, 13, (1984).

3. G. I. Dimov, A. A. Ivanov, and G. V. Roslyakov, Fiz. Plasmy, 8970 (1982) [Sov. J. Plasma Phys. (Engl. Transl.), 8546 (1982)].

4. A. A. Kabantsev, and S. Yu. Taskaev, "Low-frequence trist flute instability in a plasma jet," Fiz. Plasmy, 16 700 (1990) [Sov. J. Plasma Phys. (Engl. Transl.), 16 406 (1990)].

5. A. A. Kabantsev, and S. Yu. Taskaev, "Ion temperature in a plasma jet," Fiz. Plasmy, 18635 (1992) [Sov. J. Plasma Phys. (Engl. Transl.), 18331 (1992)].

6. G. I. Dimov, A. A. Kabantsev, S. V. Kuzmin, et. al., "Thermal insulation of the target plasma in the "AMBAL-YU” mirror device," Fiz. Plasmy, 19350
(1993) [Sov. J. Plasma Phys. (Engl. Transl.), 19180 (1993)].

7. T. D. Akhmetov, V. I. Davydenko, A. A. Kabantsev, et. al., "Longitudinal current in the initial plasma of the AMBAL-M open trap," Fiz. Plasmy, 24, (1998) [Plasma Phys. Reports (Engl. Transl.), 24, (1998)].

8. A. Simon, "Ambipolar diffusion in a magnetic field," Phys. Rev., 98, 317 (1955).

9. A. A. Kabantsev, V. G. Sokolov, and S. Yu. Taskaev, "Decrease in the Target Density during Neutral-Beam Injection into a Tandem Mirror," Fiz. Plasmy, 21, 775 (1995) [Plasma Phys. Reports (Engl. Transl.), 21, 735 (1995)].

10.S. Yoshikawa, and D. J. Rose, "Anomalous diffusion of a plasma across a magnetic field," Phys. Fluids, 5, 334 (1963).

11.B. B. Kadomzev, Voprosy Teorii Plasmy (Problems of Plasma Theory), Moscow: Atomizdat, 4, 314 (1964).

12.T. D. Akhmetov, V. I. Davydenko, A. A. Kabantsev, et. al., "Magnetic fluctuations in the initial plasma of the AMBAL-M open trap," Submitted to Fiz. Plasmy.

13.S. Yu. Taskaev, "Transverse current in the initial plasma periphery of the AMBAL-M open trap," Fiz. Plasmy, 25, (1999) [Plasma Phys. Reports (Engl. Transl.), 25, (1999)].

14.T. D. Akhmetov, V. I. Davydenko, and S. Yu. Taskaev, "Measurements by the use of small electrostatic analyzer of electrons," Submitted to Fiz. Plasmy.

15.B. A. Trubnikov, Voprosy Teorii Plasmy (Problems of Plasma Theory), Moscow: Atomizdat, 1, 98 (1963). 